\title{
A trans-acting suppressor restores splicing of a yeast intron with a branch point mutation
}

\author{
Joseph R. Couto, ${ }^{1}$ Joseph Tamm, ${ }^{1}$ Roy Parker, ${ }^{2}$ and Christine Guthrie \\ Department of Biochemistry and Biophysics, University of California, San Francisco, California 94143-0448 USA
}

\begin{abstract}
Splicing of introns fom Saccharomyces cerevisiae pre-mRNA requires the conserved sequence TACTAAC; the $3^{\prime}$-most A residue is utilized as the site of branch formation. We showed previously that the transcript from an actin-HIS4 gene fusion containing the mutation TACTAAC to TACTACC (designated C259) is spliced inefficiently, thereby preventing growth on the histidine precursor histidinol. By selecting for growth on histidinol, we have identified a mutant in which the splicing of the C259 transcript is increased fourfold; splicing of other mutated introns is not significantly improved. The mutant locus encodes a trans-acting suppressor. A single mutation, ma16-1, is sufficient for suppression; however, suppression is maximized in heterozygous diploids containing both rna16-1 and the wild-type allele RNA16. In addition, wild-type premRNAs (and lariat intermediates) accumulate in rna16-1 cells. We propose that the RNA16 locus encodes a component of the splicing machinery.
\end{abstract}

[Key Words: RNA splicing; trans-acting suppressor; branch point; yeast]

Received March 27, 1987; revised version accepted May 14, 1987.

The removal of group I, group II, and nuclear pre-mRNA introns is accomplished by mechanisms that share certain fundamental principles (Cech and Bass 1986). For example, each splicing process appears to occur via two sequential transesterification reactions. The most noteworthy distinctions among these splicing substrates involve the strategies used to fold the intron into a functionally active geometry. At one extreme is the autocatalytic splicing of the prototype group I intron; at least in vitro, splicing of the Tetrahymena pre-rRNA depends solely on the presence of highly conserved structural elements within the intron. In contrast, the only conserved features of nuclear mRNA introns appear to be restricted to short regions at or near the splice junctions; in metazoans, moreover, these sequences are often degenerate (Mount 1982; Green 1986). Presumably the low information content within this class of introns is compensated by the extensive participation of trans-acting factors. A major challenge in understanding nuclear premRNA splicing is thus the elucidation of the nature and the role of these trans-acting factors.

The first nucleolytic step in the splicing of nuclear pre-mRNA involves cleavage at the $5^{\prime}$ splice junction

\footnotetext{
1The order of the first two authors is arbitrary.

2Present Address: Department of Biology, University of California, San Diego, California 92093 USA.
}

and concomitant formation of a lariat intermediate (Padgett et al. 1984; Ruskin et al. 1984). In yeast, the lariats form at the $3^{\prime}$-most $\mathrm{A}$ in the sequence TACTAAC (Domdey et al. 1984; Rodriguez et al. 1984); this invariant sequence seems to be absolutely required for efficient splicing (Langford and Gallwitz 1983; Pikielny et al. 1983; Langford et al. 1984; Fouser and Friesen 1986; Vijayraghavan et al. 1986). In mammals, sequences adjacent to the site of lariat formation exhibit degeneracy; however, branching invariably occurs at an adenosine residue. Splicing is completed by cleavage at the $3^{\prime}$ splice junction and concomitant exon ligation, to release the intron in a lariat form and mature mRNA.

The earliest events in the pathway involve the assembly of the precursor into a large multicomponent complex referred to as the spliceosome (Brody and Abelson 1985; Frendewey and Keller 1985; Grabowski et al. 1985). Data from mammalian systems suggest that the abundant class of small nuclear ribonucleoprotein particles (snRNPs) U1, U2, U5, and U4-U6 (Maniatis and Reed 1987) play a major role in organizing the spliceosome via various snRNP : intron (Mount et al. 1983; Black et al. 1985; Chabot et al. 1985; Zhuang and Weiner 1986) and (presumably) snRNP : snRNP interactions (Chabot and Steitz 1987); the participation of at least one heteronuclear ( $\mathrm{hn}$ ) RNP protein is indicated /Choi et al. 1986) but currently less well understood.

Genetics is a powerful ally to the biochemical dissec- 
tion of complex cellular processes, particularly in cases involving multicomponent assemblies (for review, see Botstein and Maurer 1982). A successful route to the identification of interacting components has been to isolate extragenic suppressors of mutations that affect the process of interest. With this rationale in mind, several years ago we constructed a genetically manipulable splicing substrate; yeast cells containing an actin-HIS4 gene fusion can grow on the histidine precursor histidinol only if the 309-nucleotide actin intron is efficiently and accurately spliced (Parker and Guthrie 1985). The effects of point mutations introduced into the fusion intron can be scored by both biological and biochemical analyses. In this way, a number of mutations that inhibit splicing have been identified (Parker and Guthrie 1985; Vijayraghavan et al. 1986); these mutations cause growth defects on histidinol, which correlate with the accumulation of unspliced fusion transcripts. Thus, we are now in the position to search for extragenic suppressors that restore splicing of these altered fusion transcripts.

Here we describe a reversion study of the C259 mutation (Vijayraghavan et al. 1986), which alters the branch point nucleotide within the TACTAAC box (TACTAAC to TACTACC). Among cells that had spontaneously regained the ability to grow on histidinol, we identified a mutant that fulfilled a set of criteria demanded for the possession of an authentic trans-acting suppressor of the splicing defect. On the basis of the properties of this suppressor, we suggest that we have identified a structural gene encoding a component of the splicing machinery. This component might interact with the branch nucleotide.

\section{Results}

\section{Isolation of trans-acting suppressors}

An $\mathrm{A} \rightarrow \mathrm{C}$ transversion at the branch point of the actinHIS4 gene fusion (designated C259) causes the accumulation of $80 \%$ or more of the fusion transcript as unspliced pre-mRNA in vivo (Vijayraghavan et al. 1986). A $20 \%$ level of mature mRNA is insufficient to allow growth on histidinol, thus, restoration of growth on histidinol provides a powerful selection for suppressors. Some extragenic revertants are expected to arise by alterations in trans-acting factors that improve splicing of the C259 transcript.

We employed diploids in the selection process for two reasons. First, to allow the recovery of recessive-lethal suppressors, and second, because dominant suppressors, which are more likely to arise in diploids, generally confer a gain of function. The relevant genotype of the diploid parent used to select revertants is shown in Table 1. The strain is homozygous for the C259 actinHIS4 fusion, integrated at the substantially deleted HIS4 locus.

To facilitate the identification of revertants that are good candidates for further analysis, we developed a multistep protocol (explained in detail in Materials and methods). Briefly, in the first step, independent domi-
Table 1. Genotype of C259 parent

\begin{tabular}{|c|c|}
\hline Genotype & Purpose \\
\hline his4-401/his4-401 & $\begin{array}{l}\text { deletes HIS4 } \\
\text { fusion integration site }\end{array}$ \\
\hline ura3-52/ura3-52 & $\begin{array}{l}\text { maintenance of the C259- } \\
\text { actin-HIS4 integrated } \\
\text { fusion }\end{array}$ \\
\hline $\operatorname{trp} 1-1 / \operatorname{trp} 1-1$ & $\begin{array}{l}\text { maintenance of the actin- } \\
\text { lac } Z \text { plasmids }\end{array}$ \\
\hline HOL $1 / H O L 1$ & import of histidinol \\
\hline $\begin{array}{l}\text { URA3 C259-actin-HIS4/ } \\
\text { URA3 C259-actin-HIS4 }\end{array}$ & selection of revertants \\
\hline $\begin{array}{l}\text { pYAHB-2 }\{\text { C259 actin-lacZ } \\
\text { CENIII TRP1 ARS1) }\end{array}$ & $\begin{array}{l}\text { screen for trans-acting } \\
\text { suppressors }\end{array}$ \\
\hline $\begin{array}{c}\text { pADIVS ( } \triangle \text { IVS actin-lacZ } \\
\text { CENIII TRP1 ARS1) }\end{array}$ & $\begin{array}{l}\text { screen for splicing-specific } \\
\text { suppressors }\end{array}$ \\
\hline
\end{tabular}

nant revertants of the $\mathrm{C} 259$ parent were selected by their ability to grow on histidinol media. This requires the conversion of histidinol to histidine, which is the function of the HIS4C gene product (Keesey et al. 1979). In the second step, extragenic revertants were identified by assaying the initial revertants for their ability to suppress the phenotype of a second independent C259 mutation in the intron of an actin-lac $Z$ gene fusion carried on the centromere plasmid pYAHB-2 (Vijayraghavan et al. 1986). Because splicing of the lacZ fusion transcript occurs at low efficiency in the C259 parent, this strain is faint blue on X-Gal plates; revertants of interest are substantially darker in color.

Finally, to restrict our attention to trans-acting suppressors implicated in splicing per se, we included a third screen. The centromere plasmid (pYAHB-2) was replaced with a nearly identical construct (pADIVS) in which the actin intron has been deleted precisely (Larson et al. 1983); thus, $\beta$-galactosidase activity does not require splicing of the fusion transcript from pA $\Delta$ IVS. Any suppressor whose function is specific for intron removal should not affect the expression of an RNA lacking an intron. Thus, mutants that enhanced lacZ production from non-intervening sequence (IVS)containing RNA were discarded.

Using this protocol we selected 521 independent revertants. Of these, only one, R8, satisfied both of the preceding criteria for a trans-acting, splicing-specific suppressor of the defect caused by the C259 mutation.

Splicing of the C259 pre-mRNA substrate is enhanced in the $R 8$ revertant

Biochemical analyses were required to verify that the biological phenotypes reflected the restoration of efficient splicing of the C259 substrate. We prepared poly $(\mathrm{A})^{+}$ RNA from the revertant R8, the C259 parent, and a control strain (WT) that carries a wild-type actin-HIS4 fusion. Figure 1A shows a primer extension analysis using an oligonucleotide primer that is specific for fusion transcripts and allows the detection of pre-mRNA, mRNA, and the lariat intermediate. In WT (lane 3), pre-mRNA is essentially undetectable, while in the C259 parent (lane 

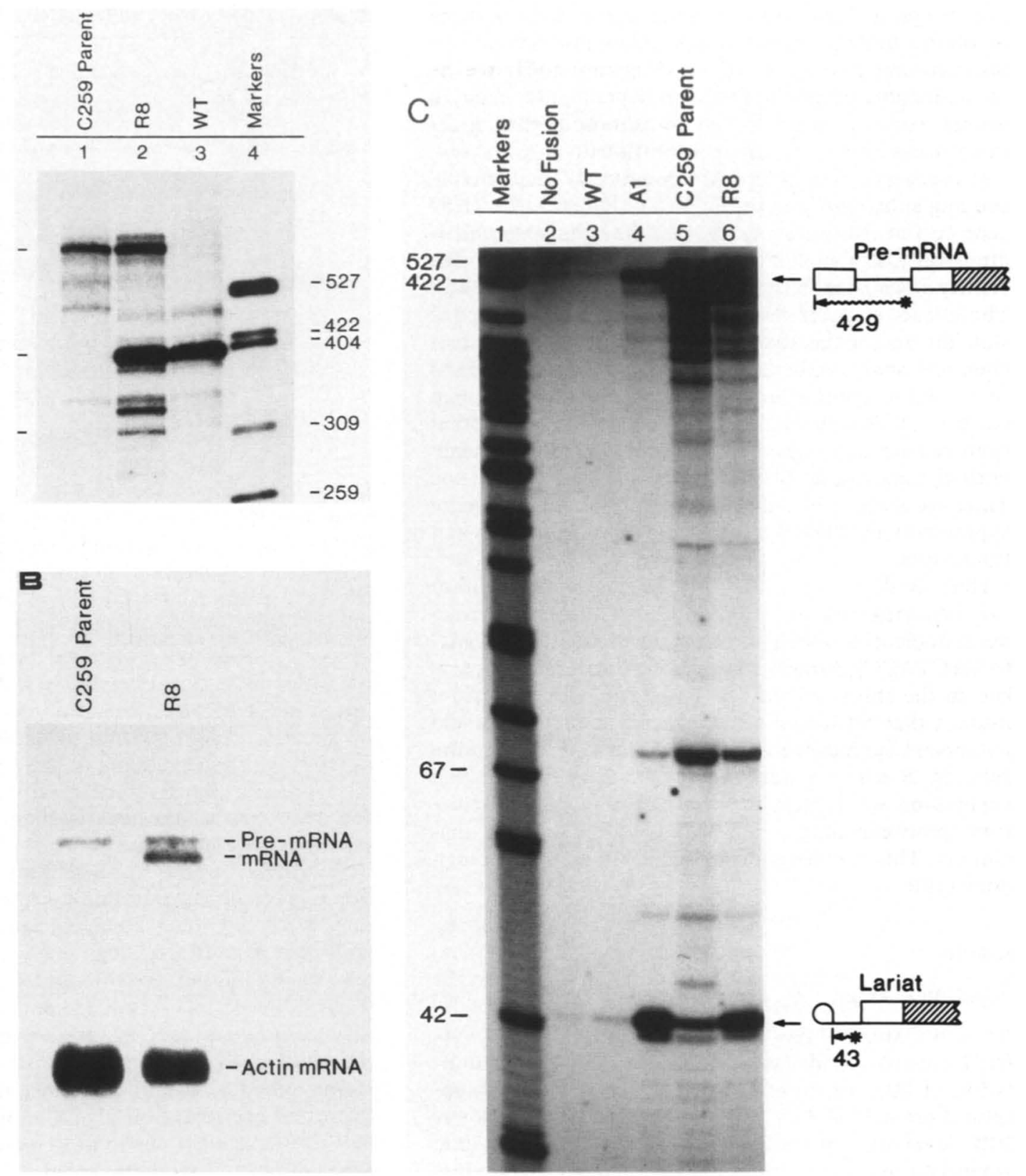

Figure 1. RNA analysis of R8. Poly $(A)^{+}$RNA was extracted from the strains indicated (grown in YEPD) and analyzed by primer extension $(A$ and $C)$ or Northern hybridization $(B)$. The following oligonucleotides were used (see Materials and methods): $(A)$ the $19^{\prime}$-mer, which hybridizes the HIS4 sequences at the actin-HIS4 junction, including 2 nucleotides of actin, $(B)$ the $21^{\prime}$-mer, which hybridizes to the actin $3^{\prime}$ exon, and $(C)$ the $24^{\prime}$-mer which hybridizes to the $3^{\prime}$ end of the actin intron. Size markers are $5^{\prime}$-end-labeled, HpaII-cut pBR325. (WT) $39 / 59$ containing a wild-type actin-HIS4 fusion; (Al) FC8-24D with an Al actin-HIS4 fusion /used here to mark the branch point); (No fusion) 39/59, a strain with no actin-HIS4 gene fusions. In the diagrams (not drawn to scale) the position of the oligonucleotide annealing is indicated by * ; actin exons are open boxes, HIS4 sequences are slashed boxes, the actin intron is a solid line, and cDNAs are wavy lines. The numbers indicate the length of the cDNAs. The first band migrating above the lariat band in lane $2(A)$ is not reproducible in other RNA preparations. The band immediately above it is most likely caused by a stable hairpin in pre-mRNA (Parker and Patterson 1987). In the Northern (B), fusion lariat intermediates run just above mRNA and are not clearly resolved. In lane $2(C)$, lariat and pre-mRNA cDNA products are detected in the absence of the fusion due to the presence of the actin gene. In lanes 4,5 , and $6(C)$, the band at approximately 70 nucleotides is most likely the same stop caused by the stable hairpin noted in $A$.

1) more than $90 \%$ of the fusion transcript accumulates as mRNA precursor. In contrast, R8 (lane 2) accumulates approximately $40 \%$ pre-mRNA with a concomitant increase in the levels of mRNA and lariat intermediate. To confirm these results, we performed the Northern analysis shown in Figure 1B; again we see that more than half the transcripts in $\mathrm{R} 8$ are spliced RNA. Note that suppression is indicated by a change in the ratio of pre- 
mRNA to mRNA (and intermediates); the absolute amounts of RNA are not germane to the suppressing effect because they depend on the number of gene fusions present in the particular strain. Specifically, R8 shows a decrease in the relative amount of pre-mRNA (lpremRNA//pre-mRNA + lariat + mRNA)].

In Figure 1A, the band corresponding to the lariat intermediate is significantly darker in R8 than in the C259 parent. This is a reproducible finding (Figs. 2, 3, and 5, and other data not shown). To demonstrate that the primer extension block characteristic of the lariat species occurs at the same nucleotide in $\mathrm{R} 8$ as in the C259 parent, we performed a primer extension analysis using an oligonucleotide primer whose $3^{\prime}$ terminus is 43 nucleotides downstream of the branch point. As shown in Figure 1C, the R8 block coincides precisely with that of the parent; as we demonstrated previously /Vijayraghavan et al. 1986), the lariat forms at the position of the mutated $\mathrm{C} 259$ residue (TACTACC). In principle, these lariats may be dead-end products, while the functional intermediates branch at an alternative site and are converted rapidly to mRNA. We consider this possibility unlikely, however. As shown in lane 3, lariat intermediates are detectable by this assay even in wild-type substrates. Almost certainly, a lariat formed at an alternative branch point should accumulate at least as much, if not more, than do wild-type species, and thus be detected in our analysis. We conclude that R8 suppression does not occur by selection of an alternative branch point, but rather by more efficient processing using the mutated site.

\section{The suppressor is encoded by a single genetic locus}

If the suppressor in $\mathrm{R} 8$ is due to a mutation at a single genetic locus, then it should determine a phenotype that segregates $2: 2$ in tetrads. It is possible to follow the phenotype of suppression itself in tetrads because R8 diploids yield four viable meiotic products; i.e., the suppressor is not lethal. The analysis of suppression is most easily done by scoring growth on histidinol medium. This has been done for eight tetrads /see Materials and methods) and allows us to conclude that growth on histidinol segregates $2: 2$. To confirm that suppression occurs at the level of splicing, we analyzed RNA patterns directly. Total RNA was extracted from two tetrads grown in medium lacking uracil (-Ura) and analyzed by primer extension, using the primer employed in Figure 1A. The analysis of tetrad JI1-1 is shown in Figure 2. Spores $\mathrm{A}$ and $\mathrm{C}$ grow on histidinol and show an increase in the splicing efficiency, as measured by the relative amounts of each RNA species (see Fig. 2 legend for densitometric scanning values). Note, however, that splicing is considerably less efficient in either spore $\mathrm{A}$ or spore C than in the parent R8 diploid (see Fig. 1A). An explanation for the observation that suppression is submaximal in haploid cells is the subject of a later section. The analysis of the second tetrad (JJ1-2) gave similar results (not shown). We conclude that suppression of the splicing defect exhibits a $2: 2$ segregation, and is therefore determined by a mutation at a single genetic locus.

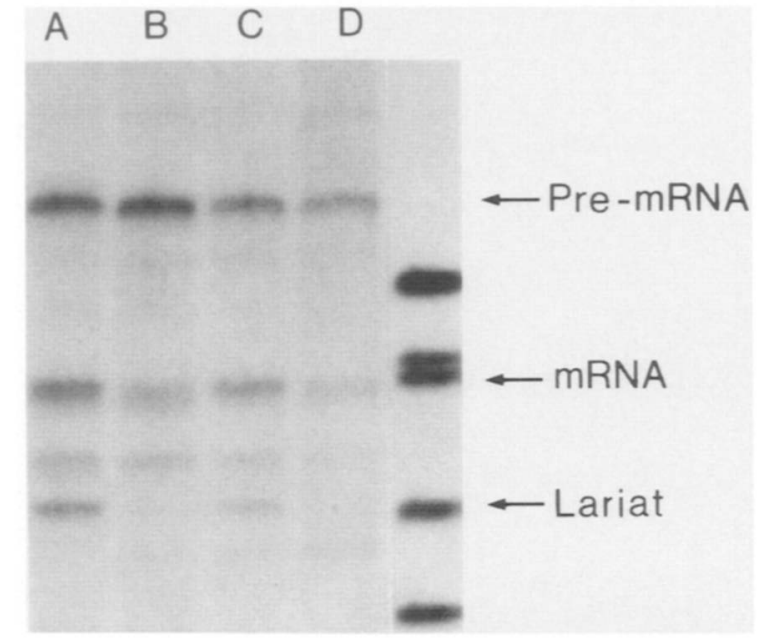

Figure 2. Primer extension analysis of an R8 tetrad. R8 was sporulated and tetrads were dissected. Tetrad JJl-1 was grown in synthetic medium lacking uracil (-Ura) and total RNA was extracted from each haploid. The RNAs were analyzed by primer extension using the oligonucleotide 19'-mer (see Fig. 1A). The film was traced to determine the percentages of premRNA, mRNA, and lariat. They are, respectively: lane $A(48$, $34,18)$, lane $B(79,19,2)$, lane $C(59,30,11)$, and $D(75,21,4)$.

\section{The suppressor is extragenic}

The preceding section does not address the possibility that suppression is caused by a second site mutation within the actin-HIS4 gene fusion. To demonstrate that the suppressor is extragenic, we first mated a haploid that exhibited biochemical suppression (JJ1-2D) to a wild-type haploid that does not contain an actin-HIS4 gene fusion (JJ5-31B) (see Materials and methods); the resulting diploid was sporulated and dissected. The segregation of the actin-HIS4 fusion was scored by assaying the ability of the spores to grow on synthetic media lacking uracil (Table 1). As shown in Figure 3, haploids that grow are scored as "+" for the fusion. Since only half the spores are expected to receive the fusion, and since the fusion is necessary to determine the segregation of the suppressor, we crossed each of the meiotic products to an otherwise wild-type strain that carries the C259 actin-HIS4 fusion (see legend to Fig. 3).

Poly $(\mathrm{A})^{+}$RNA was extracted from each of the resulting diploids and assayed by primer extension. If a diploid contains the suppressor it accumulates approximately $40 \%$ of the fusion transcript as precursor and is scored as "+" in Figure 3; diploids lacking the suppressor accumulate mainly $(80 \%)$ full-length fusion precursor and are scored as "-". As in Figure 2, we see a $2: 2$ segregation of biochemical suppression. Most importantly, these results demonstrate that suppression of the C259 splicing defect segregates independently of the actin-HIS4 gene fusion derived from R8 and is, therefore, extragenic. We have designated the suppressor mutation rna16-1. This nomenclature was chosen because other mutations affecting RNA splicing also have the 


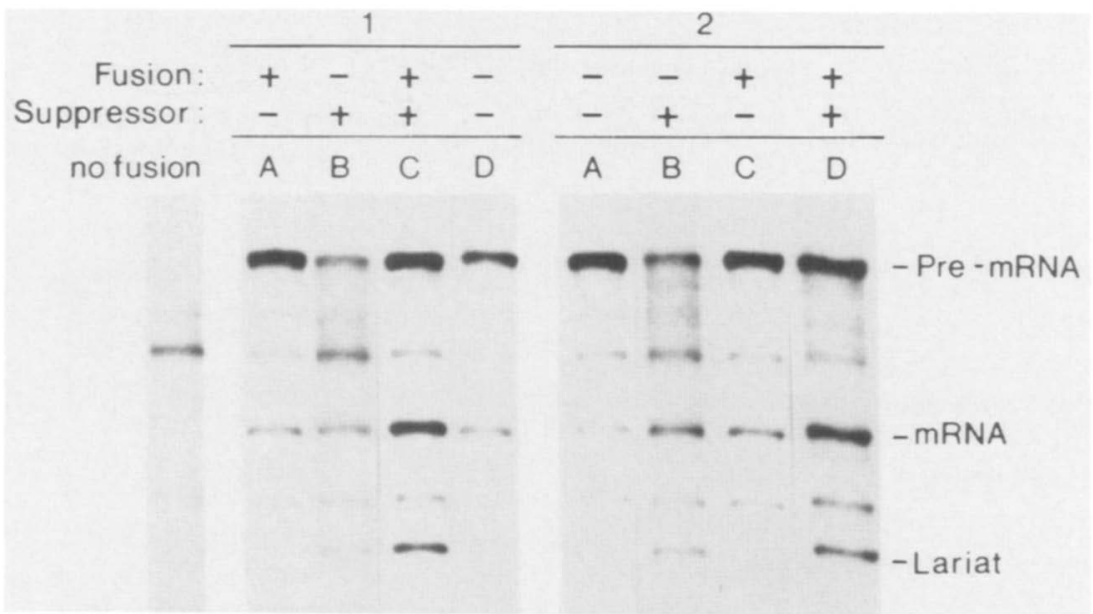

Figure 3. Suppressor linkage analysis. The two tetrads shown here were obtained as described in the text. To score for the presence of the fusion we tested the haploids on synthetic medium lacking uracil (- Ura plates) and scored growth as indicated after "Fusion"; the scores were confirmed with Southern analysis (not shown). The haploids were then mated to either YJC39 or YJC59, depending on the mating type; both mating strains contained C259 actin-HIS4 fusions. Poly(A)+ RNA from the resulting diploids (grown in YEPD) was analyzed by primer extension using the oligonucleotide $19^{\prime}$-mer (see Fig. 1A). Suppression was scored on the basis of the pre-mRNA/ (pre-mRNA + mRNA + lariat) ratio. "No Fusion" is 39/59, a strain with no actin-HIS4 fusions. Differences in the total RNA levels in different lanes are attributable to differences in the number of gene fusions.

RNA designation (Rosbash et al. 1981; Larkin and Woolford 1983; Teem et al. 1983; Miller 1984; Lustig et al. 1986).

\section{The suppressor is specific for the C259 mutation}

It is important to determine if the suppressor interacts with a specific region of the intron, i.e., does it suppress only the intron mutation that it was selected for, or can it also restore the splicing of various mutated introns? A specific suppressor will potentially provide more information about the mechanism of splicing. Thus, we asked whether ma16-1 could suppress additional mutations in the intron of the actin-HIS4 fusion (Vijayraghavan et al. 1986). The mutations tested include alterations at two other positions within the TACTAAC box. TAATAAC (A256) and TACAAAC (A257), shown in Figure 4; two mutations at the $5^{\prime}$ junction, /ATATGT (A1) and /CTATGT (C1) (not shown); and a mutation at the 3' splice junction $\mathrm{AC} / \mathrm{ac}$ (C303/305) (not shown). With the possible exception of the A256 case, the presence of the suppressor has no appreciable effect on the relative amounts of pre-mRNA, lariat, and mRNA. It is difficult to rule out completely suppression in the case of A256 because this mutation already allows a considerable amount of pre-mRNA $(50-70 \%)$ to be processed completely. Nonetheless, it is clear that the level of A256 mRNA observed in the presence of the suppressor is not increased appreciably.

We can thus conclude that the only dramatic effect observed in our tests is the suppression of the defect caused by the C259 mutation. Therefore, the suppressor exhibits specificity with respect to functionally important sites within the actin intron. It will be of interest to determine whether the phenotypes of branch point mutations other than C259 can also be suppressed.

\section{The wild-type RNA16 allele increases the efficiency of} suppression

We observed (Table 2) that when RNA for primer extension analysis is prepared from strains cultured in rich medium (YEPD), suppression is observed in the R8 diploid and in diploids derived from it, but not in its haploid meiotic products. (The data shown in Fig. 2 were ob-

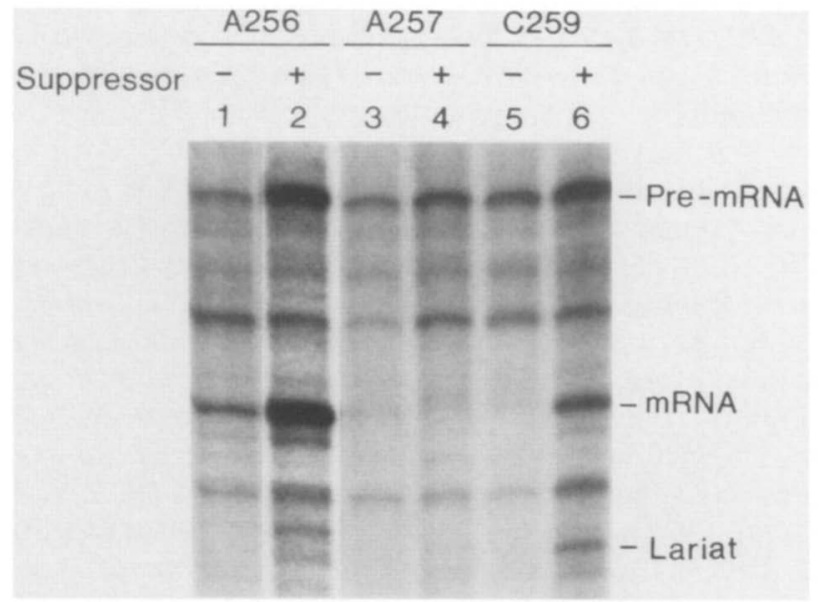

Figure 4. Suppressor specificity. Primer extensions were performed on total RNA prepared from the diploids described below (grown in - Ura synthetic medium) using the $19^{\prime}$-mer oligonucleotide. Parent diploids (odd lanes) are RNA16/RNA16 diploids that were made as described for C259 parent (see Materials and methods) except that appropriately mutated pYAH-I2 replaced C259-mutated pYAH-I2. Suppressor diploids (even lanes) are RNA16/ma16-1 diploids that were obtained by first mating haploids JJ16-3D and JJ5-31B /see Materials and methods|, and then transforming with appropriately mutated pYAH-I2. 
Table 2. Relative amounts of RNA species

\begin{tabular}{lrrrrrrr}
\hline & \multicolumn{3}{c}{ Rich medium $^{\mathrm{a}}$} & & \multicolumn{3}{c}{ Synthetic medium } \\
\cline { 2 - 4 } & $\% \mathrm{P}$ & $\% \mathrm{M}$ & $\% \mathrm{~L}$ & & $\% \mathrm{P}$ & $\% \mathrm{M}$ & $\% \mathrm{~L}$ \\
\hline RNA16 & 92 & 7 & 1 & 77 & 20 & 3 \\
RNA16/RNA16 & 83 & 14 & 3 & 82 & 14 & 4 \\
ma16-1 & 84 & 13 & 3 & 53 & 32 & 15 \\
ma16-1/rna16-1 & 87 & 8 & 5 & 45 & 39 & 16 \\
RNA16/rna16-1 & 41 & 34 & 25 & 22 & 58 & 20 \\
\hline
\end{tabular}

These values were obtained by quantitating densitometric tracings of typical primer-extension analyses (including those shown in Figs. 2 and $5 B$ ).

a(P) pre-mRNA; (M) mRNA; (L) lariat.

tained from cells grown in synthetic media; see below.) To test the hypothesis that, in cells grown in rich medium, suppression requires heterozygosity at the RNA16 locus, we constructed and analyzed by primer extension a series of heterozygous and homozygous diploids, as described in Figure 5A and summarized in Table 2. Homozygous wild-type diploids (lanes 1 and 2), as expected, accumulate primarily unspliced C259 fusion transcript (approximately 85\%). Three independent rna16-1/ RNA16 heterozygotes (lanes 3, 4, and 5) show the expected increase in mRNA splicing and accumulate only $40 \%$ pre-mRNA. In contrast, each of three diploids homozygous for ma16-1 (lanes 6, 7, and 8) show a splicing pattern essentially indistinguishable from that of the homozygous wild-type. These results were confirmed by Northern analysis (not shown). We conclude that, during growth in rich medium, measurable suppression of the splicing defect requires both the RNA16 and ma16-1 alleles.

We then asked if the presence of the RNA16 allele affected the degree of suppression when strains are cultured in synthetic media. RNA prepared from each of the three diploid types was analyzed by primer extension, and the results compared to those shown in Figure $5 \mathrm{~A}$ by densitometric tracing of the autoradiograms. As shown in Figure $5 \mathrm{~B}$, and in Table 2, splicing is almost undetectable in rna16-1/rna16-1 cells grown in rich medium (Fig. 5B, panel F), but is very efficient when these cells are cultured in synthetic medium (panel C). In fact, the relative amounts of pre-mRNA, mRNA, and lariat in homozygotes grown in synthetic medium (panel $\mathrm{C}$ ) are similar to those of rna16-1/RNA16 heterozygotes grown in rich medium (panel E). Thus, under certain growth conditions, efficient suppression does not require both alleles. On the other hand, the efficiency of splicing in rna16-1/RNA16 heterozygotes grown in synthetic medium (panel B) is even greater than that seen for the ma16-1/ma16-1 homozygotes grown under the same conditions (panel C). The relative amounts of mature fusion RNA from cells cultured in synthetic medium (panels A, B, and C) correlate with the relative growth rates of the same cells on histidinol plates /which are synthetic); RNA16/rna16-1 diploids grow well, rna16-1/ ma16-1 diploids grow poorly, and RNA16/RNA16 diploids do not grow (not shown). We conclude that the presence of the RNA16 allele significantly increases the efficiency of suppression in all growth conditions.
Cells homozygous for mal6-1 accumulate precursors of wild-type transcripts

If RNA16 encodes a spliceosome component, it is reasonable to expect that wild-type transcripts would be spliced less efficiently in rna16-1 mutants. To test this hypothesis, we first analyzed the splicing of two such transcripts in RNA16/RNA16, RNA16/rna16-1, and rna16-1/rna16-1 diploids. As shown in Figure 6A, the splicing of the MATa1 transcript is significantly affected in mutant homozygous diploids (lanes 6, 7, and 8); fulllength precursor and partially spliced intermediates accumulate dramatically at the expense of mature RNA. Splicing efficiency in the heterozygous diploid (lanes 3, 4 , and 5) is essentially indistinguishable from that in the homozygous wild-type strain (lanes 1 and 2). Analysis of a second spliced transcript, rp51A (Fig. 6B), shows that unspliced pre-mRNA, which is undetectable in wildtype (lanes 1 and 2) or heterozygous (lanes 3-5) strains also accumulates in rna16-1/rna16-1 cells (lanes 6-8).

To extend these observations to haploids, we performed primer extension analysis of these wild-type transcripts in the two tetrads examined previously (JJ1-1, JI-2). As predicted, rp51A pre-mRNA accumulates in each of the two spores containing the rna16-1 allele (data not shown). Similarly, as shown in Figure 7, the ma16-1 spores containing the MATa mating type allele accumulate unspliced MATa1 pre-mRNA and intermediates.

We conclude that rna16-1 determines two distinct phenotypes: (1) the dominant suppression of the splicing defect caused by the $\mathrm{C} 259$ mutation; and (2) the recessive decrease in the splicing efficiency of pre-mRNAs containing wild-type introns.

\section{Discussion}

The suppressor has the properties expected for an altered component of the splicing machinery

We have described the isolation in diploids of a suppressor that enhances the splicing of the mutant C259actin-HIS4 transcript fourfold in vivo (see Table 2). The suppressor mutation, designated ma16-1, is genetically unlinked to the parental C259 gene fusion, and segregates $2: 2$ in tetrads. Therefore, suppression occurs because of a modification in a single, trans-acting factor. It is unlikely that this trans-acting factor affects either the transcription or the stability of the fusion RNA since the revertant $\mathrm{R} 8$ shows no difference in actin-lacZ expression, relative to the parent strain, when this fusion lacks the actin IVS. Moreover, the suppressor is dominant, which makes it unlikely to affect RNA stability. In addition to suppressing the splicing defect of C259 actinHIS4 mutant transcripts, the trans-acting factor causes an increase in the amount of unspliced wild-type premRNAs; this phenotype is recessive. These two independent splicing phenotypes argue strongly that the trans-acting factor is directly involved in the splicing of nuclear pre-mRNAs. 


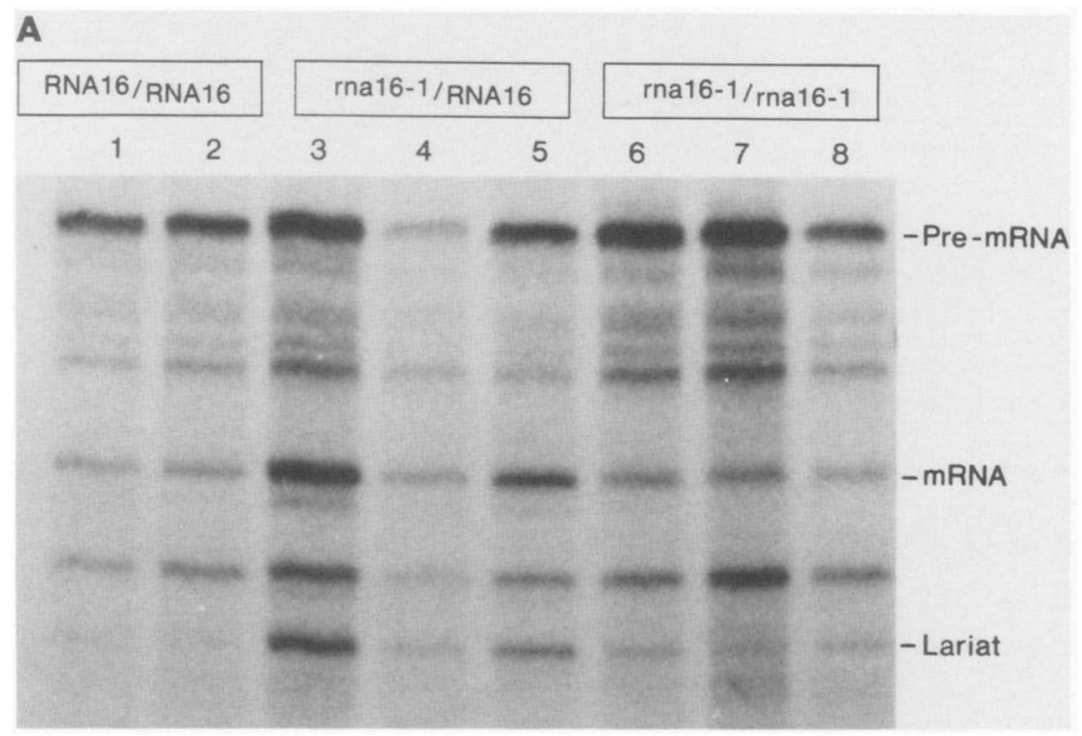

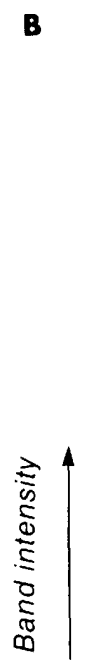

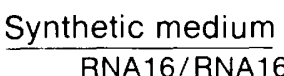

RNA16/RNA16

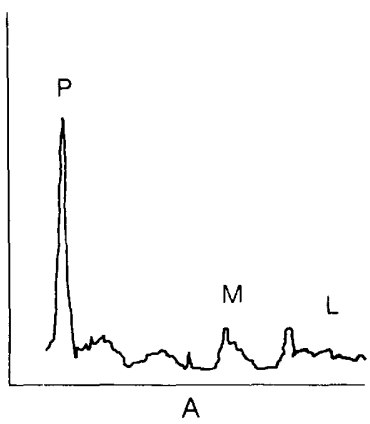

rna16-1/RNA16

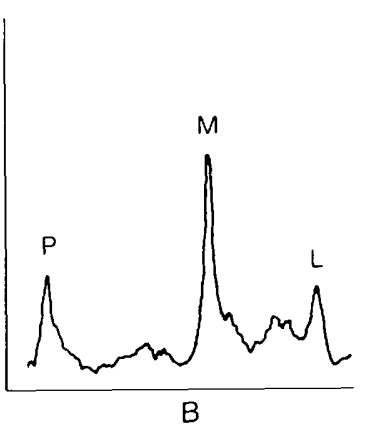

Rich medium RNA16/RNA16

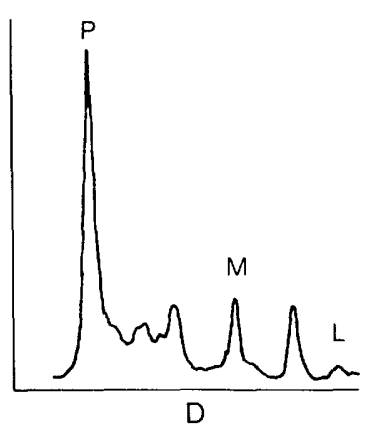

rna16-1/RNA16

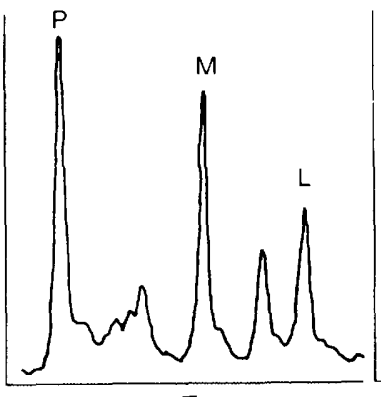

E

rna16-1/rna16-1

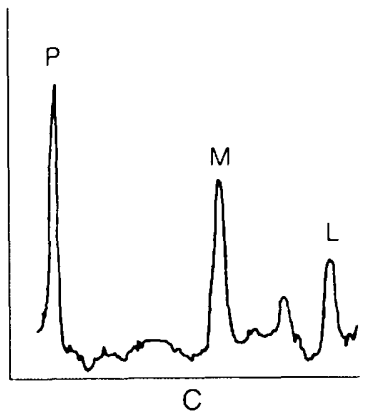

rna16-1/rna16-1

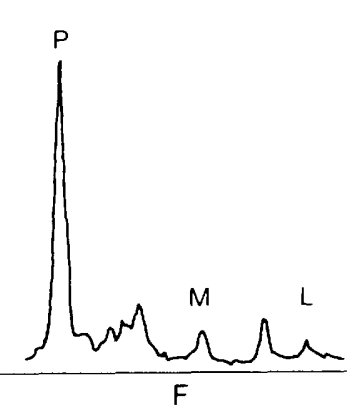

Top $(-) \longrightarrow$ Bottom(+)

Figure 5. (A) Primer extension analysis of diploids grown in rich medium (YEPD). Poly $(\mathrm{A})^{+}$RNAs were analyzed by primer extension using the oligonucleotide 19'-mer (see Fig. 1A). The different kinds of diploids were obtained as follows. First, an rna16-1 haploid (JJ1-2D) containing the C259 gene fusion was mated to a wild-type haploid (JJ5-31B). The heterozygous diploids were then sporulated and dissected, and two tetrads ( 1 and 2$)$ were analyzed for the presence of the fusion and for the segregation of $r n a 16-1$. This analysis is the subject of Fig. 3. The two RNA16/RNA16 diploids were obtained by mating $1 \mathrm{~A} \times \mathrm{YJC59}$ and $2 \mathrm{~A} \times \mathrm{YJC} 39$, respectively. The rna16-1/RNA16 diploids were obtained by mating haploids $1 \mathrm{C} \times \mathrm{YJC} 39,2 \mathrm{~B} \times \mathrm{YJC59}$, and 2D $\times$ YJC39. Both YJC39 and YJC59 contained C259 actin-HIS4 gene fusions. The ma16-1/rna16-1 diploids were obtained by crossing the haploids $1 \mathrm{~B} \times 1 \mathrm{C}, 1 \mathrm{C} \times 2 \mathrm{~B}$, and $2 \mathrm{~B} \times 2 \mathrm{D}$, respectively. $(B)$ The effect of the growth media upon suppression. $(D, E$, and $F)$ Densitometric tracings of a lighter exposure of lanes 1,3, and 6 of Fig. 5A. These same diploids were grown in synthetic medium lacking uracil (-Ura); total RNA was extracted and analyzed by primer extension using the oligonucleotide 19'-mer. Scans of light exposures of these primer extensions are shown in $A, B$, and $C$. 


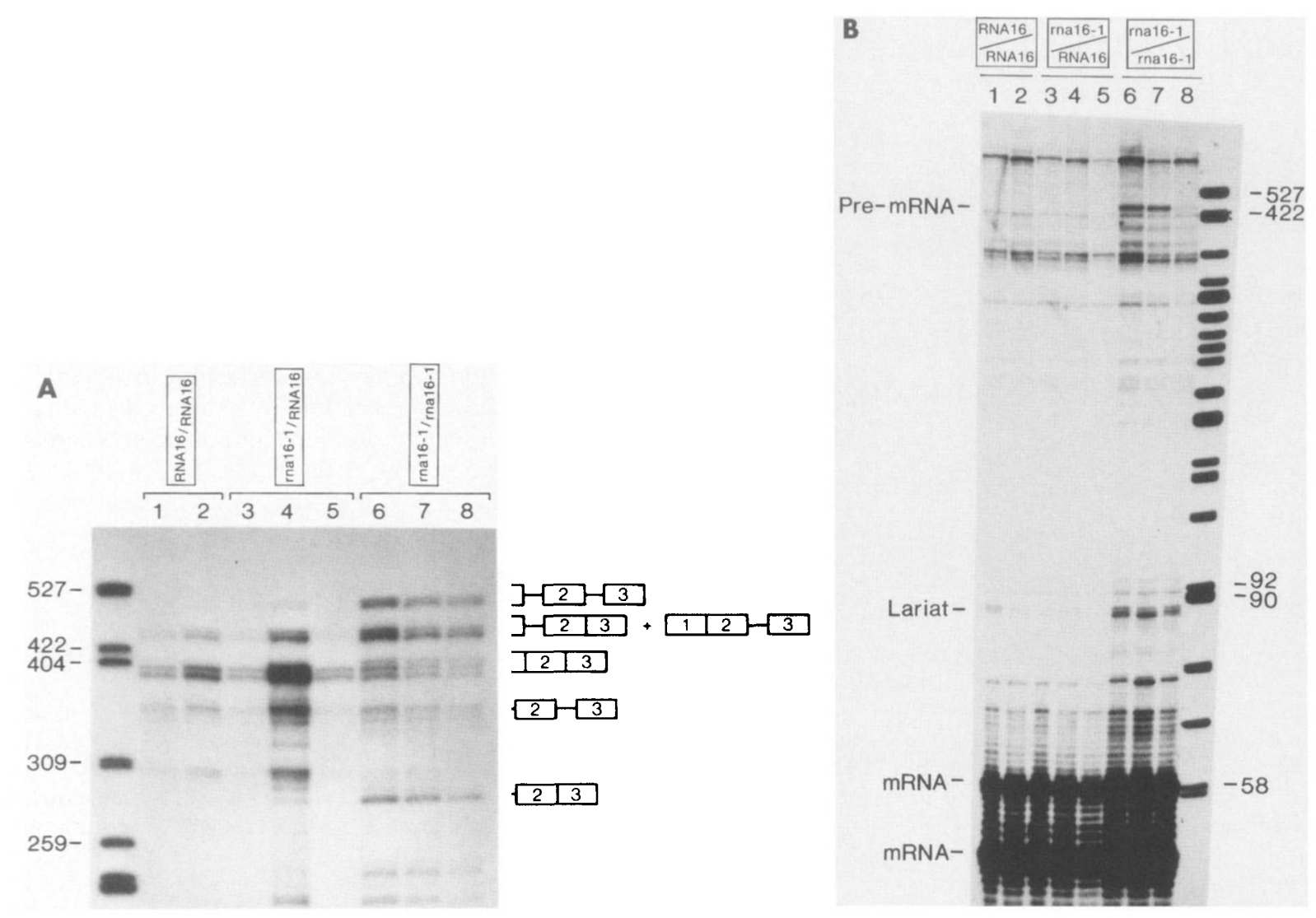

Figure 6. Primer extension analysis of wild-type mRNAs in homozygous diploids. The strains analyzed here are the same as those shown in Fig. 5A; all cells were grown in rich medium (YEPD). Poly(A) ${ }^{+}$RNA was analyzed using either the MATa1 oligonucleotide $(A)$ or an rp51A oligonucleotide, RB1 (B). The RNA species were identified on the basis of electrophoretic mobility. The doublets in $A$ are reproducible and probably represent two transcription initiation sites. In $B$, the two mRNA bands represent two different RNA polymerase starts. Overexposure of the gel in $B$ did not reveal an appreciable accumulation of rp5lA pre-mRNA in lanes 1-5. Conversely an underexposure still allowed detection of pre-mRNA in lanes 6-8. In the diagrams (not drawn to scale), exons are open boxes and introns are solid lines.

The suppressor acts early in the splicing pathway

Certain mutations at the $5^{\prime}$ and $3^{\prime}$ splice sites [such as A1 and C303/305 (Vijayraghavan et al. 1986)] result in the accumulation of lariats. In contrast, the C259 mutation causes the accumulation of pre-mRNA but not of lariat intermediates. Therefore, C259 must disrupt one or more steps in the splicing pathway that precede lariat formation. Since the rna16-1 gene product suppresses the C259 defect, resulting in a decreased ratio of premRNA to mRNA, it must also (at least) act prior to lariat formation.

Concomitantly with the decrease in the relative level of C259 pre-mRNA, the relative level of lariat increases in the presence of the suppressor. Possibly, then, the ma16-1 gene product also inhibits the conversion of lariat to mRNA. In support of this hypothesis, the relative levels of MATa1 and rp51 lariats also seem to increase in rna16-1/rna16-1 diploids (this is less apparent in haploids). One explanation for these observations is that while the presence of the rna16-1 gene product in the splicing complex mainly affects the conversion of pre-mRNA to lariat, it might also affect other steps indi- rectly, by causing a physical distortion of the spliceosome.

The RNAl6 factor is likely to recognize the TACTAAC region

The suppressor has no apparent effect on the splicing of $\mathrm{Al}, \mathrm{Cl}, \mathrm{A} 257$, or $\mathrm{C} 303 / 305$ transcripts and has little or no effect on the splicing of the A256 transcript (cf. Vijayraghavan et al. 1986). Since the C1, A257, and C303/305 mutations are phenotypically tight, a slight effect of the suppressor on the splicing of these substrates might not be detectable. In contrast, the $\mathrm{Al}$ and the A256 mutations, like C259, both block the conversion of pre-mRNA to lariat, and both are phenotypically leaky; yet, the Al mutation is not suppressed at all, and the A256 mutation is, at best, suppressed only marginally. We conclude, therefore, that the suppressor is likely to be allele specific. This preference for the site of branch formation is indicative of a physical contact between the suppressor molecule and the TACTAAC box.

Our laboratory has recently demonstrated a basepairing interaction between a yeast U2-like RNA (Ares 


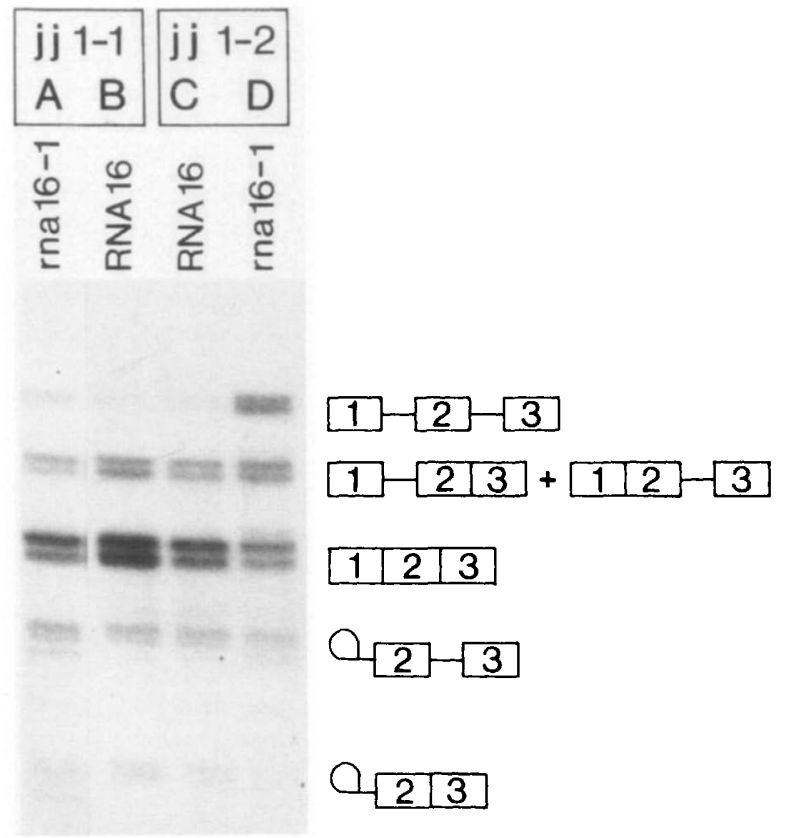

Figure 7. Primer extension analysis of wild-type mRNAs in haploids. Total RNA was extracted from MATa haploids of tetrads JJ1-1 and J/1-2 and analyzed by primer extension using the MATa1 oligonucleotide.

et al. 1986) and the TACTAAC box (Parker et al. 1987). It is likely that this complementarity mediates an early association between the U2-like snRNP and the site of subsequent branch formation. Interestingly, in the proposed scheme, the branch point nucleotide is not itself paired, and it is suggested that recognition of the branch nucleotide occurs subsequent to this Watson-Crick interaction. The RNA16 gene product is obviously an excellent candidate for this function. Recent results make it unlikely that RNA16 is this snRNA (snR20 in our nomenclature; Riedel et al. 1986), since $s n R 20$, when encoded in a centromere plasmid and transformed into an ma16-1 haploid, was unable to relieve the ma16 $^{-}$recessive defect on the splicing of the MATa1 transcript.

Cloning of the RNA16 and rna16-1 alleles is in progress. The recessive defect conferred by ma16-1 on splicing of wild-type transcripts suggests to us that a null allele will be lethal. If this is the case, it should then be possible to identify gene products that interact with the RNA16 gene product by the isolation of suppressors of rna16 mutations with conditional phenotypes. In particular, these experiments should reveal the relationship between RNA16-and the gene products with which it interacts-and the rna2-rna11 mutations, which produce splicing defects both in vivo (Rosbash et al. 1981; Larkin and Woolford 1983; Teem et al. 1983; Miller 1984) and (at least in some cases) in vitro (Lustig et al. 1986).

The clones will also help in our understanding of the following two observations that make the rna16-1 suppressor unique. Suppression of the C259 defect is most efficient in heterozygous diploids, i.e., when both the wild-type and mutant alleles are present. In addition, suppression is enhanced when cells are grown in synthetic medium (see Table 2). One model that addresses both observations is that, in rna16-1 haploids, wild-type pre-mRNAs act as competitive inhibitors of suppression, since the rna16-1 gene product must splice both the $\mathrm{C} 259$ and the wild-type transcripts. In heterozygous diploids, the participation of the RNA16 gene product in the splicing of wild-type pre-mRNAs would allow a higher effective concentration of the rna16-1 gene product to splice the $\mathrm{C} 259$ transcripts. The media effect would be explained if the concentration of wild-type pre-mRNAs is disproportionally increased in rich medium. If this model is correct, then the expression of the rna16-1 clone by a multicopy plasmid in ma16-1 haploids should improve suppression, especially in rich medium.

\section{Materials and methods \\ Plasmids}

The plasmid pYAH-I2 contains the actin-HIS4 gene fusion and was constructed by Parker and Guthrie (1985). The TACTAAG sequence, 14 nucleotides upstream of the TACTAAC box, is deleted from the actin intron except in A256 (see below). The TACTAAC box is present either in its wild-type form or mutated as follows: C259-TACTACC, A256-TAATAAC, and A257-TACAAAC (Vijayraghavan et al. 1986). Likewise, the $5^{\prime}$ (/GTATGT) and $3^{\prime}$ (AG/) consensus sequences are mutated in the appropriate versions of the plasmid: Al-/ATATGT, Cl-/ CTATGT, and C303/305-AC_/ac (Vijayraghavan et al. 1986). The plasmid pYAHB-2 contains an actin-lacZ gene fusion and is described in Vijayraghavan et al. (1986); pA $\Delta$ IVS is similar to pYAHB-2 but it has a precise deletion of the actin intron (Larson et al. 1983).

\section{Strains}

YJC39: (MATo RNA16 ade5 trp1-1 his4-401 leu2 ura3-52 can1 HOL1). YJC59: (MATa RNA16 ade2 trp1-1 his4-401 leu2 ura352 can1 HOL1). JJ5-25C: (MATa RNA16 ade5 his4-401 leu2

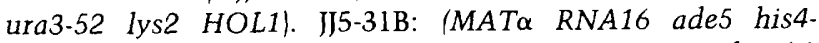
401 leu2 ura3-52 lys2 HOL1). FC8-24D containing the Al actin-HIS4 fusion was used to map the branch point as described in Vijayraghavan et al. (1986). The diploid 39/59 was made by mating YJC39 with YJC59. The diploid "C259 Parent" was made as follows: YJC39 and YJC59 were each transformed with XbaI-cut C259-mutated pYAH-I2; YJC39 and YIC59 transformants were selected on - Ura plates and subsequently mated one to the other; correct integration of the plasmid into the his 4-401 locus was confirmed by Southern analysis of the resulting diploids; they were then transformed with C259-mutated pYAHB-2. IJ1-2D is a haploid from an R8 tetrad. Its genotype is MATa rna16-1 ade2 trp1-1 his4-401 leu2 ura3-52 HOL1, and it contains the C259 actin-HIS4 fusion (with URA3) integrated at the his4-401 locus. JJ16-3D is a haploid with the following genotype: MATa ma16-1 his4-401 trp11 leu2 ura3-52 HOL1 ade-.

\section{Media}

YEPD, - Ura, and - Trp (synthetic media lacking uracil or tryptophan) are described in Sherman et al. (1974). Histidinol 
medium is described in Parker and Guthrie (1985); X-Gal plates are described in Rose et al. (1981).

\section{Yeast genetic methods}

All yeast genetic methods, including matings, sporulation, tetrad dissection, phenotypic analysis, replica-plating with velvets and with a 48-point inoculator, and lithium acetate transformations were performed as described in Sherman et al. (1974).

\section{Isolation of the $R 8$ revertant}

Ten thousand colonies of the C259 parent growing on YEPD plates (about 200/plate) were replica-plated onto histidinol plates and incubated at $30^{\circ} \mathrm{C}$. A total of 521 spontaneous papillae, each arising from an independent colony, were picked after 5 days. Each of these isolates and the C259 parent were transferred with a 48-point inoculator to X-Gal plates to monitor expression of the $\mathrm{C} 259$-mutated actin-lacZ fusion from pYAHB-2. After incubating $3-4$ days at $30^{\circ} \mathrm{C}$, the color intensity of the revertants was compared with that of the parent. Sixteen clones exhibited a darker blue color than the parent and were chosen for further study. The 16 clones were cured of their $C E N-A R S$ plasmid (pYAHB-2) by growing them overnight in YEPD. Plasmid loss was monitored by scoring growth on YEPD and - Trp plates. Cells that had lost pYAHB-2 were retransformed with the plasmid pA $\Delta$ IVS. Transformants were tested on X-Gal as before and their color intensity was compared with that of the C259 parent (without pYAHB-2) transformed with pA $\Delta$ IVS. Only one revertant, R8, was equal in color to the C259 control. The remaining 15 were darker and were discarded.

\section{Phenotypic analysis of R8 tetrads}

The diploid revertant $\mathrm{R} 8$ was sporulated and tetrads were dissected. Eight complete tetrads were analyzed for the presence of the suppressor by scoring growth on histidinol plates. Four growth phenotypes were observed. Very strong growth and normal growth were scored + . Leaky growth was very slow growth that was not reproducible, i.e., sometimes the same haploid would grow poorly and sometimes not at all. This phenotype and reproducible nongrowth were both scored - . Seven of the eight tetrads showed a $2: 2,+:-$ segregation and one a $3: 1,+:-$ segregation; $20 / 39$ spores from additional dissections (which included incomplete tetrads) scored + .

We believe the reason for the differences in growth rates is as follows: R8 has acquired an extra copy of actin-HIS 4 gene fusion so that one chromosome contains two gene fusions and the other contains one. This was confirmed by Southern analysis and probably resulted from recombination between flanking direct repeats in different chromatids. The segregation of the gene fusions among R8 tetrads is then $2: 2: 1: 1$. This was also confirmed by Southern analysis across three tetrads (JJ1-1, -2, and $-4 \mid$. Very strong growth is observed when a spore has two gene fusions and the suppressor. Normal growth is observed when a spore has one fusion and the suppressor. Leaky growth may occur if the spore has two gene fusions and no suppressor.

\section{RNA analysis}

Total RNA was prepared as described by Domdey et al. (1984). Poly(A) + RNA was prepared as follows. Total RNA was dissolved in $4.5 \mathrm{ml}$ of low-salt buffer [ $10 \mathrm{~mm}$ Tris- $\mathrm{HCl}(\mathrm{pH} 7.4)$, $0.2 \% \mathrm{SDS}, 1 \mathrm{mM}$ EDTA], incubated at $65^{\circ} \mathrm{C}$ for $3 \mathrm{~min}$, and quickly cooled on ice. $\mathrm{NaCl}$ was added to a concentration of 0.5 $\mathrm{M}$ and the RNA was mixed with $100 \mathrm{mg}$ of oligo(dT)-cellulose, type 3 (Collaborative Research Inc.), previously equilibrated in high-salt buffer $[0.5 \mathrm{M} \mathrm{NaCl}, 10 \mathrm{~mm}$ Tris- $\mathrm{HCl}(\mathrm{pH} 7.4), 0.2 \%$ SDS, $5 \mathrm{~mm}$ EDTA]. The mixture was gently rocked for $40 \mathrm{~min}$ in a $15-\mathrm{ml}$ tube at $23^{\circ} \mathrm{C}$. Following centrifugation, the supernatant, containing poly $(A)^{-}$RNA, was removed. The resin was washed five times with $5 \mathrm{ml}$ of high-salt buffer, at $23^{\circ} \mathrm{C}$, each time rocking for $3 \mathrm{~min}$ and pelleting the resin by centrifugation. Poly $(\mathrm{A})+$ RNA was eluted in two successive 5-min washes each with $2.5 \mathrm{ml}$ of low-salt buffer at $23^{\circ} \mathrm{C}$. The eluates were combined and ethanol-precipitated. The RNA was resuspended in $50 \mu \mathrm{l}$ of water.

Northern analysis: RNA was resolved on glyoxal gels as described in McMaster and Carmichael (1977), blotted to GeneScreen, and probed with end-labeled oligonucleotides.

Primer and primer extensions: The oligonucleotides 19'-mer, and $24^{\prime}$-mer are described in Vijayraghavan et al. (1986); the 21'-mer (CCAGAACCGTTATCAATAACC) hybridizes to exon 2 of actin. RBl is described in Teem and Rosbash (1983) and was a generous gift from Dr. Michael Rosbash. The MATa1 oligonucleotide (GAATTTATTTAGATCTCATACGTTT) hybridizes to exon 3 of the MATa1 transcript. Primer extensions were performed as described by Domdey et al. (1984).

Southern analyses were performed basically as described in Sherman et al. (1974). Genomic DNA was digested with PstI, and the fragments were resolved on $0.8 \%$ agarose gels, transferred to nitrocellulose, and probed with a nick-translated HIS4 clone.

Densitometric tracings: Light exposures on Kodak Diagnostic Film, X-Omat AR, were scanned with a Zeineh Soft Laser scanning densitometer (LKB) using the laser beam. Quantitation was done by cutting and weighing photocopies of the tracings.

\section{Acknowledgments}

We thank Lucita Esperas for excellent technical assistance, Judy Piccini for help in the preparation of the manuscript, and Aaron Mitchell, Bob Sauer, Linda Couto, and the members of the Guthrie laboratory for critical reading of the manuscript and helpful discussions. J.T. and J.C. were supported by postdoctoral fellowships from the National Institutes of Health. This work was supported by grants GM21119 from the National Institutes of Health and DCB-8603926 from the National Science Foundation to C.G.

\section{References}

Ares, M. 1986. U2 RNA from yeast is unexpectedly large and contains homology to vertebrate $\mathrm{U} 4, \mathrm{U} 5$, and $\mathrm{U} 6$ small nuclear RNAs. Cell 47: 49-59.

Black, D.L., B. Chabot, and J.A. Steitz. 1985. U2 as well as U1 small nuclear ribonucleoproteins are involved in pre-messenger RNA splicing. Cell 42: 737-750.

Botstein, D. and R. Maurer. 1982. Genetic approaches to the analysis of microbial development. Annu. Rev. Genet. 16: 61-83.

Brody, E. and J. Abelson. 1985. The "spliceosome": Yeast premessenger RNA associates with a $40 \mathrm{~S}$ complex in a splicing-dependent reaction. Science 228: 963-967.

Cech, T.R. and B.L. Bass. 1986. Biological catalysis in RNA. Annu. Rev. Biochem. 55: 599-629.

Chabot, B. and J.A. Steitz. 1987. Multiple interactions between the splicing substrate and small nuclear ribonucleoproteins in spliceosomes. Mol. Cell. Biol. 7: 281-293.

Chabot, B., D.L. Black, D.M. LeMaster, and J.A. Steitz. 1985. The 3' splice site of pre-messenger RNA is recognized by a small nuclear ribonucleoprotein. Science 230: 1344-1349. 
Choi, Y.D., P.J. Grabowski, P.A. Sharp, and G. Dreyfuss. 1986. Heterogeneous nuclear ribonucleoproteins: Role in RNA splicing. Science 231: 1534-1539.

Domdey, H., B. Apostol, R.J. Lin, A. Newman, E. Brody, and J. Abelson. 1984. Lariat structures are in vivo intermediates in yeast pre-mRNA splicing. Cell 39: 611-621.

Fouser, L.A. and J.D. Friesen. 1986. Mutations in a yeast intron demonstrate the importance of specific conserved nucleotides for the two stages of nuclear mRNA splicing. Cell 45: $81-93$.

Frendewey, D. and W. Keller. 1985. Stepwise assembly of a premRNA splicing complex requires U-snRNPs and specific intron sequences. Cell 42: 355-367.

Grabowski, P.J., S.R. Seiler, and P.A. Sharp. 1985. A multicomponent complex is involved in the splicing of a messenger RNA precursor. Cell 42: 345-353.

Green, M.R. 1986. Pre-mRNA splicing. Annu. Rev. Genet. 20: $671-708$.

Keesey, J.K., R. Bigelis, and G.R. Fink. 1979. The product of the HIS4 gene cluster in Saccharomyces cerevisiae. I. Biol. Chem. 254: 7427-7433.

Langford, C.J. and D. Gallwitz. 1983. Evidence for an introncontained sequence required for the splicing of yeast RNA polymerase II transcripts. Cell 33: 519-527.

Langford, C.J., F.-J. Klinz, C. Donath, and D. Gallwitz. 1984. Point mutations identify the conserved, intron-contained TACTAAC box as an essential splicing signal sequence in yeast. Cell 36: 645-653.

Larkin, J.C. and J.L. Woolford. 1983. Molecular cloning and analysis of the CRY1 gene: A yeast ribosomal protein gene. Nucleic Acids Res. 11: 403-420.

Larson, G.P., K. Itakura, H. Ito, and I.J. Rossi. 1983. Saccharomyces cerevisiae actin-Escherichia coli lacZ gene fusions: Synthetic-oligonucleotide-mediated deletion of the 309 base pair intervening sequence in the actin gene. Gene 22: $31-$ 39.

Lustig, A.J., R.-J. Lin, and J. Abelson. 1986. The yeast RNA gene products are essential for mRNA splicing in vitro. Cell 47: 953-963.

Maniatis, T. and R. Reed. 1987. The role of small nuclear ribonucleoprotein particles in pre-mRNA splicing. Nature 325: $673-678$.

McMaster, G.K. and G.G. Carmichael. 1977. Analysis of singleand double-stranded nucleic acids on polyacrylamide and agarose gels by using glyoxal and acridine orange. Proc. Natl. Acad. Sci. 74: 4835-4838.

Miller, A.M. 1984. The yeast MATa1 gene contains two introns. EMBO J. 3: 1061-1065.

Mount, S.M. 1982. A catalogue of splice junction sequences. Nucleic Acids Res. 10: 459-472.

Mount, S.M., I. Pettersson, M. Hinterberger, A. Karmas, and J.A. Steitz. 1983. The U1 small nuclear RNA-protein complex selectively binds a $5^{\prime}$ splice site in vitro. Cell 33: 509518.

Padgett, R.A., M.M. Konarska, P.J. Grabowski, S.F. Hardy, and P.A. Sharp. 1984. Lariat RNA's as intermediates and products in the splicing of messenger RNA precursors. Science 225: 898-903

Parker, R. and C. Guthrie. 1985. A point mutation in the conserved hexanucleotide at a yeast $5^{\prime}$ splice junction uncouples recognition, cleavage, and ligation. Cell 41: 107118.

Parker, R. and B. Patterson. 1987. Architecture of fungal introns: Implications for spliceosome assembly. In Molecular biology of RNA: New perspectives (ed. B. Dudock). Academic Press, Orlando, Florida. (In press).

Parker, R., P. Siliciano, and C. Guthrie. 1987. Recognition of the TACTAAC box during mRNA splicing in yeast involves base-pairing to the U2-like snRNA. Cell 49: 229-239.

Pikielny, C.W., J.L. Teem, and M. Rosbash. 1983. Evidence for the biochemical role of an internal sequence in yeast nuclear mRNA introns: Implications for UI RNA and metazoan mRNA splicing. Cell 34: 395-403.

Riedel, N., J.A. Wise, H. Swerdlow, A. Mak, and C. Guthrie. 1986. Small nuclear RNAs from Saccharomyces cerevisiae: Unexpected diversity in abundance, size, and molecular complexity. Proc. Natl. Acad. Sci. 83: 8097-9001.

Rodriguez, J.R., C.W. Pikielny, and M. Rosbash. 1984. In vivo characterization of yeast mRNA processing intermediates. Cell 39: 603-610.

Rosbash, M., P.K.W. Harris, J.L. Woolford, and J.L. Teem. 1981. The effect of temperature-sensitive RNA mutants on the transcription products from cloned ribosomal protein genes of yeast. Cell 24: 679-686.

Rose, M., M.J. Casadaban, and D. Botstein. 1981. Yeast genes fused to $\beta$-galactosidase in Escherichia coli can be expressed normally in yeast. Proc. Natl. Acad. Sci. 78: 2460-2464.

Ruskin, B., A.R. Krainer, T. Maniatis, and M.R. Green. 1984. Excision of an intact intron as a novel lariat structure during pre-mRNA splicing in vitro. Cell 38: 317-331.

Sherman, F., G.R. Fink, and C.W. Lawrence. 1974, revised annually. Methods in yeast genetics. Cold Spring Harbor Laboratory, Cold Spring Harbor, New York.

Teem, I.L. and M. Rosbash. 1983. Expression of a $\beta$-galactosidase gene containing the ribosomal protein 51 intron is sensitive to the ma2 mutation of yeast. Proc. Natl. Acad. Sci. 80: $4403-4407$.

Teem, J.L., J.R. Rodriguez, L. Tung, and M. Rosbash. 1983. The rna2 mutation of yeast affects the processing of actin mRNA as well as ribosomal protein mRNAs. Mol. Gen. Genet. 192: $101-103$.

Vijayraghavan, U., R. Parker, J. Tamm, Y. Iimura, J. Rossi, J. Abelson, and C. Guthrie. 1986. Mutations in conserved intron signals affect multiple steps in the yeast splicing pathway, particularly assembly of the spliceosome. EMBO J. 5: $1683-1695$.

Zhuang, Y. and A.M. Weiner. 1986. A compensatory base change in Ul snRNA suppresses a $5^{\prime}$ splice site mutation. Cell 46: 827-835. 


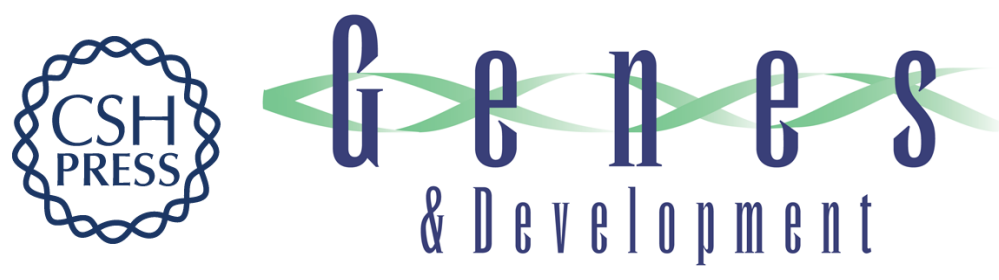

\section{A trans-acting suppressor restores splicing of a yeast intron with a branch point mutation.}

J R Couto, J Tamm, R Parker, et al.

Genes Dev. 1987, 1:

Access the most recent version at doi:10.1101/gad.1.5.445

References This article cites 37 articles, 10 of which can be accessed free at:

http://genesdev.cshlp.org/content/1/5/445.full.html\#ref-list-1

License

Email Alerting Receive free email alerts when new articles cite this article - sign up in the box at Service the top right corner of the article or click here.

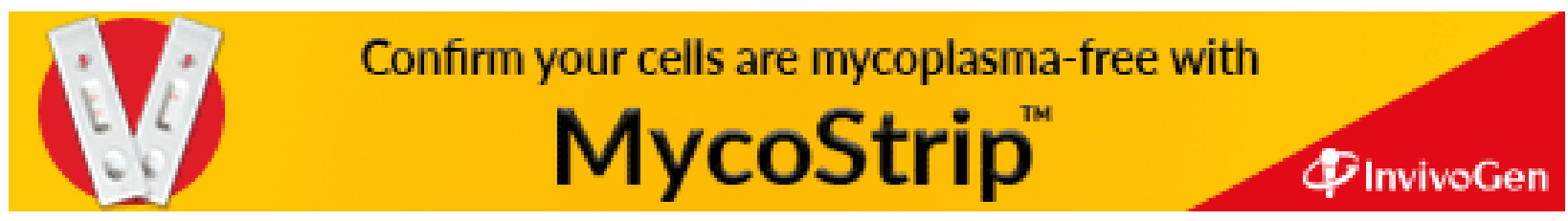

